RESEARCH ARTICLE

\title{
The influence of health insurance enrolment on maternal and childcare use in Nigeria
}

\author{
Rolle Remi Ahuru $^{1,2^{*}}$ Daniel Osaze ${ }^{2}$ Akpojubaro Henry Efegbere ${ }^{3}$ \\ ${ }^{1}$ Department of Economics, Faculty of Social Sciences, University of Benin, Benin City, Nigeria \\ ${ }^{2}$ Center of Excellence in Reproductive Health Innovation, University of Benin, Benin City, Nigeria \\ ${ }^{3}$ Department of Community Medicine, College of Medical Sciences, Edo University Iyamho, Edo State, Nigeria
}

\section{Check for updates}

Correspondence to: Rolle Remi Ahuru, Department of Economics, Faculty of Social Sciences, University of Benin, Benin City, Nigeria \& Center of Excellence in Reproductive Health Innovation, University of Benin, Benin City, Nigeria;

E-mail: remirolle1986@yahoo.com

Received: November 13, 2020;

Accepted: January 3, 2021;

Published: January 5, 2021

Citation: Ahuru RR, Osaze D and Efegbere AH. The influence of health insurance enrolment on maternal and childcare use in Nigeria. Soc Work Soc Welf, 2021, 2(1): 82-90.

https://doi.org/10.25082/SWSW.2021.01.001

Copyright: (C) 2020 Rolle Remi Ahuru, et al. This is an open access article distributed under the terms of the Creative Commons Attribution License, which permits unrestricted use, distribution, and reproduction in any medium, provided the original author and source are credited.

\begin{abstract}
Purpose: Health insurance reduces the cost of using modern maternal and child cares and encourages women to use modern care services. This is because health insurance scheme spread the burden of maternal care usage across people and overtime. In Nigeria, there is a dearth of research evidence on the effect of health insurance enrolment on maternal and childcare use. This study examined the effect of health insurance coverage on maternal and childcare use in Nigeria, drawing upon data from the most recent National Demographic and Health Survey (2018). Methods: Three outcome indicators were used: a minimum of four antenatal care (ANC) visits, place of delivery, and complete child immunization. Descriptive and predictive analytical methods were utilized. A representative sample of 33,715 women who reported recent birth within the last five years preceding the Survey was used for the analyses. Analyses were undertaken using STATA version 13.0 for windows. Results: The results showed that $57 \%$ of the women made a minimum of four ANC visits, $41 \%$ delivered in health institutions, and $27 \%$ undertook complete child immunization. Enrolment in health insurance was low as only $2.3 \%$ of the women were under any form of health insurance coverage. However, enrolment in health insurance significantly improves the odds for a minimum of four ANC visits [aOR: $1.52, \mathrm{p}=0.00$ ] and health facility delivery [aOR: $1.42, \mathrm{p}=0.00$ ]. However, there is no significant difference in complete child immunization between women who were under health insurance and those who were not [aOR: 1.36, $\mathrm{p}=0.28$ ]. Also, residing in an urban area, Southern geopolitical zones, and being drawn from wealthy homes confer an advantage on women to use modern maternal and child healthcare. Conclusion: Pragmatic interventions should be initiated to encourage women's enrolment in health insurance in Nigeria. Communitybased health insurance scheme should be encouraged among rural women and those of them in the informal sector.
\end{abstract}

Keywords: influence, health insurance, maternal, childcare, Nigeria

\section{Introduction}

Globally, maternal and child mortality rates have fallen since 1990. For instance, maternal deaths fell from 471,000 in 2000 to 295,000 in 2017 [1]. In sub-Saharan Africa (SSA), maternal mortality rate fell by $45 \%$ between 1990 and 2015, though unequally across the countries [2]. The success is largely attributed to the various interventions programmes sponsored by the Millennium Development Goals (MDGs), and the various national and international health development organizations. The understanding that maternal mortality is the primary cause of death among women within the reproductive ages accounts for the motivation of both intensive research and funding allocation in the sphere of reproductive health for improving both provision and utilization of modern maternal care services. Despite the efforts, progress remained below optimum. Evidence from the World Health Organization [1] puts the annual number of maternal mortality at 295,000. A recent Nigerian study reported daily maternal mortality of 800 [3]. Still, over $99 \%$ of maternal mortality is accounted for by low middle-income countries (LMICs), showing inequality in access to health services and structural barriers limiting access to maternal care services among women in LMICs.

Maternal Health Service constitutes an essential component of Maternal and Child Health $(\mathrm{MCH})$ services and its key objective is to guarantee good health for pregnant women throughout the entire pregnancy cycle [4]. Maternal Health Services encapsulate preconception care, antenatal care (ANC), delivery care, postnatal care and family planning services. The overriding 
objective why women are advised to go for ANC checkups is to track the progress of their pregnancy and put in place measures to avert complications in case of potential risks. Childbearing has been described as a journey that holds both risk and uncertainty for most women in LMICs. This has been the situation despite medical advancements that have made pregnancy a risk-free exercise for all mothers [5], through a set of strategies that have proved to be efficacious in saving the lives of mothers even in several poor countries [4]. The WHO [6] recommends access to a continuum of maternal care services as key intervention programmes that can meaningfully reduce maternal and perinatal mortality among developing countries. However, many women in LMICs do not utilize modern care services due to socioeconomic, cultural and health system-related barriers [2].

Past Nigerian studies reported the underlying barriers to maternal care utilization $[7,8]$. From a behavioural perspective, maternal care behaviour is significantly influenced by financial constraints and geographical distance to health facilities. Several demand and supply-side interventions have been proposed and implemented with the intent to improve women's access to modern maternal care services [9], which include community education and teachings, use of community outreach services, conditional cash transfer scheme, free maternal care services, and health insurance schemes in Asia and Africa [2]. However, the influence of health insurance scheme on maternal and childcare use has not been extensively researched in Nigeria, hence constraining conclusive findings.

An extensive number of studies reported a positive impact of health insurance enrolment on maternal care utilization $[2,10,11]$. In Nigeria, several persons are not under health insurance coverage. For instance, evidence shows that over $90 \%$ of Nigerians are not under any form of health insurance coverage [12]. Nigerian government initiated the National Health Insurance Scheme (NHIS) as an integral aspect of its health policy in the 1990s with full implementation in2005; designed to facilitate the achievement of the health-related Millennium Development Goals, particularly goal 4 and 5.The Federal government launched the NHIS to reduce catastrophic expenditures associated with healthcare-related costs. In Nigeria, NHIS caters for ANC services, caesarean and postnatal care services. There has been an increase in the utilization of both out-patient and in-patient healthcare following the introduction of NHIS in 2005, yet there is a dearth of detailed evidence on whether women's enrolment in NHIS has positively influenced their access to modern care. However, Dahiru \& Ochei [10] in a study less focused on the role of health insurance on maternal care utilization revealed that health insurance enrolment significantly improves access to maternal care services. While this study may be argued to be old [Dahiru and Ochei (2015) draw their data from National Demographic and Health Survey (2013), but this study draw its data from the most recent Demographic and Health Survey (2018)], it did not investigate the impact of health insurance enrolment on childcare utilization. Against this backdrop, the study drew upon data from the most recent National Demographic and Health Survey (2018) to explore the effects of health insurance enrolment, and selected socio-demographic factors on maternal and childcare utilization for Nigeria.

\section{Methods}

\subsection{Data source}

The data analyzed in this study were drawn from the most recent National Demographic and Health Survey [13]. The NDHS [13] is the sixth edition of the Survey since the exercise started in 1990. Demographic and Health Survey represents a nationally representative sample on health information. It uses a two-stage sampling procedure to elicit information from individuals in their houses. In the first stage, enumeration areas (EAs) are selected from the master sampling frame. In the second stage households in the enlisted EAs are counted and recruited for the survey. NDHS [13] enlisted 1,400 EAs across the country. The Survey utilized three questionnaires: household, women and men. The data analyzed in this study were extracted from the women's Survey which provided information on women's reproductive health issues such as family planning, fertility, birth spacing, child mortality, women's healthcare, childcare, abortion, breastfeeding practices and children nutritional status. The Survey was principally funded by USAID in collaboration with other agencies. The ICF provided technical support. It was implemented by national population commission in conjunction with the National Malaria Elimination Programme of the Federal Ministry of Health. NDHS [13] has two advantages over previous NDHS. First, Computer Assisted Personal Interviewing (CAPI) was used in collecting the data and this fast-tracked the collection of data and also enhanced data collection. Second, larger sample size was collected. In all 127,545 women within the reproductive ages were interviewed. Out of these women, 33,715 reported recent birth in the last five years preceding 
the Survey. The analyses in this study are bounded for this set of women.

\subsection{Outcome measures}

This study was inspired by the need to empirically investigate the impact of health insurance enrolment on maternal and childcare use among Nigerian women. Also, the effect of sociodemographic factors on maternal and childcare was examined. Three independent variables were adopted. The indicators were a minimum of four ANC (MANC) visits, place of delivery (POD) and full child immunization (CCIM). Our definition of a minimum of four ANC visits was drawn from focused ANC, which requires a woman to make a minimum of four ANC visits during the pregnancy circle. A minimum of four ANC visits was binary, hence respondents who reported they made at least four ANC visits in their most recent pregnancies were coded 1 , while those who made less than four together with those who reported no visits were coded 0 . The outcome indicator place of delivery investigated where respondents gave birth to their last child Place of delivery was recoded into a binary variable, hence respondents who reported facility deliveries were coded 1 while those who reported non-facility deliveries were coded 0 . For full child immunization we adopted the definition of Ahuru [14], which defined complete child immunization as a dose of Bacillus Calmetric Guerin (BCG); 3 doses of Diphtheria Pertussis and Tetanus [DPT], 3 doses of polio and a dose of measles within the first year of birth. Respondents who reported complete child immunization were coded 1 , while those who did not were coded 0 .

\subsection{Independent variables}

In this study, we regressed the three outcome indicators on health insurance enrolment(treatment variable) while controlling for eight socio-demographic variables: maternal age (Age), maternal education (EDUC), household wealth index (HWI), region (REG), place of residence (RES), employment status (ES), sex of household head (SHH) and financial inclusion (FICC). All independent variables were redefined into two categories to suit a binary logistic methodology adopted for this study. For maternal age, we considered two age groups $(15 \mathrm{y}-34 \mathrm{y})$ years and (35y-49y) years. Women within the age brackets $(15 y-34 y)$ were coded 0 and those within the age group (35y-49y) year were coded 1. For maternal education, NDHS [13] provided four categories: no formal education, primary, secondary and higher. We recoded maternal education into two. We lumped respondents who reported primary, secondary and higher education and called formal education, hence we considered formal education and no formal education. Respondents who reported formal education were coded 1 while those without formal education were coded 0 . Place of residence considered whether respondents were drawn from the rural or urban part of Nigeria. We coded respondents from rural part 0 while those from urban parts were coded 1. Region considered whether respondents were drawn from the Southern or Northern part of Nigeria. We lumped all the Northern geopolitical zones and called Northern region and all Southern geopolitical zones were lumped together and called the Southern region. Women from Northern regions were coded 0 while those from Southern regions were coded 1. Religion considered two options that are, Christian and others. NDHS [13] provided the following religion: Catholic, Other Christians, Islam, African traditional worshippers and others. We lumped Christian and Catholic and called them Christians and other religious groups were lumped together and called others. Christian women were coded 1 while other religion coded 0 . Household wealth index was generated using principal component analysis. The survey categorized respondents into five groups: poorest, poor, average, wealthy and wealthiest. We recode household wealth index by lumping those respondents from poorest, poor and average and called them poor, while wealthy and wealthiest were called wealthy. Women drawn from wealthy homes were coded 1 while those from poor homes were coded 0 . Employment status is binary categorizing women currently working from those not working. Working women were coded 1 while not working women coded 0 . Sex of household head categorized women into households headed by a male and those headed by a female. Women drawn from households headed by males were coded 0 while those from households headed by women were coded 1 . Financial inclusion investigated if the woman had a bank account. Those who had bank accounts were considered financially inclusive and code 1 , while those who do not were considered not financially inclusive and coded 0 .

\subsection{Statistical analysis}

Analyses for the study were undertaken at two levels and that are the univariate and multivariate levels. At the univariate level, the summary statistics for each of the variables were analyzed by showing the mean, standard deviation, minimum and maximum values. At the multivariate 
level, binary logistic regression was used to examine the relationship between health insurance coverage and the three healthcare indicators, while adjusting for other socio-demographic factors that are theoretically and empirically pertinent to the outcome indicators. The results of the logistic regression were presented as odds ratio (OR) and probability values. Statistical significance for all statistical analyses was set at 5\%. The estimated models were examined for robustness by considering the count $\mathrm{R}^{2}$ and the log-likelihood ratio [14].

\subsection{Ethical approval}

The protocol for data collection used by the Demographic Health Survey was approved by the Ethics Committee of ORC Macro INC. The study was based on analysis of anonymised secondary data hosted in the DHS public domain which absolved us of approval from respondents. However, approval for use of data was gotten from DHS.

\section{Results}

\subsection{Summary statistics}

Table 1 Summary statistics of variables

\begin{tabular}{lcccc}
\hline Variables & Mean & Std. Dev. & Maximum & Minimum \\
\hline Age & 35.8900 & 7.9400 & 49 & 15 \\
EDUC & 0.4996 & 0.5000 & 1 & 0 \\
HWI & 0.3114 & 0.4631 & 1 & 0 \\
RES & 0.3097 & 0.4624 & 1 & 0 \\
REG & 0.3452 & 0.4754 & 1 & 0 \\
ES & 0.7407 & 0.4382 & 1 & 0 \\
HIE & 0.0230 & 0.1501 & 1 & 0 \\
FICC & 0.1609 & 0.3674 & 1 & 0 \\
SHH & 0.6756 & 0.6756 & 1 & 0 \\
MANC & 0.5733 & 0.4946 & 1 & 0 \\
CCIM & 0.2732 & 0.5647 & 1 & 0 \\
POD & 0.4091 & 0.4917 & 1 & 0 \\
Soure: Auth & & & & 1 \\
\hline
\end{tabular}

Source: Authors' computation (2020)

In Table 1, the mean age of the women utilized in the study is 36 years while the spread around the mean which is the standard deviation is 8 years; hence the range for reproductive age in this study is 28 years and 44 years. Hence, women who are below 28 years are considered too young for childbearing and those above 44 years are considered too old. Approximately $57 \%$ of women in the study made a minimum of four ANC visits, $41 \%$ delivered in health institutions and $27 \%$ undertake complete child immunization. On the average, maternal utilization rate in the study is approximately $42 \%$ (that is, $57 \%+41 \%+27 \% \div 3$ ). Instructively, only $2.3 \%$ of the women reported they enrolled in health insurance.

\subsection{A minimum of four ANC visits}

In Table 2, we present the result of the influence of health insurance enrolment and other socio-demographic factors on a minimum of four ANC visits. It can be seen that all the variables exert significant influence on a minimum of four ANC visits. The model is collectively significant as shown by the log-likelihood ratio (L-R) statistic.

The count $\mathrm{R}^{2}$ was calculated for the model. The count $\mathrm{R}^{2}$ put at 0.78 (that is, $78 \%$ ) shows that the variation in health insurance enrolment and the selected socio-demographic factors in the model have correctly explained or predicted $78 \%$ of the likelihood that a pregnant woman in Nigeria will undertake a minimum of four ANC visits. Note that all the variables are binary; hence the coefficients are not interpreted in terms of marginal effects. We, therefore, turn to the odds ratios for the magnitude of the log-likelihood. From the result, we can conclude that being under health insurance enrolment confers an advantage on women to adequately utilize ANC. Women who were under health insurance coverage [aOR: $1.52, p=0.00$ ] were $52 \%$ significantly more likely to complete a minimum of four ANC visits. In reference to women who had no formal education, those who reported at least primary education [aOR: $2.70, p=$ 0.00 ] were $170 \%$ significantly more likely to undertake a minimum of four ANC visits. Women residing in rural parts of the country [aoR: $0.69, \mathrm{p}=0.00$ ] were significantly less likely to undertake a minimum of four ANC visits when compared to those from the urban parts of 
Table 2 Multivariate analysis of factors influencing maternal and child health indicators

\begin{tabular}{llll}
\hline Variables & MANC $(\mathrm{OR})$ & POD $(\mathrm{OR})$ & CCIM (OR) \\
\hline Age (ref=15y-34y) & $1.00(0.95)$ & $1.05(0.99)$ & $5.0(0.42)$ \\
EDUC (ref=no formal education) & $2.70(0.00)^{*}$ & $3.00(0.00)^{*}$ & $4.32(0.00)^{*}$ \\
HWI (ref=poor) & $1.73(0.00)^{*}$ & $1.96(0.00)^{*}$ & $4.32(0.28)$ \\
RES (ref=urban) & $0.69(0.00)^{*}$ & $0.77(0.00)^{*}$ & $0.46(0.00)^{*}$ \\
REG (ref=Northern) & $1.71(0.00)^{*}$ & $1.89(0.00)^{*}$ & $6.41(0.00)^{*}$ \\
ES (ref=not working) & $1.40(0.00)^{*}$ & $1.23(0.00)^{*}$ & $1.36(0.00)^{*}$ \\
HIE (ref=No ) & $1.52(0.00)^{*}$ & $1.42(0.00)^{*}$ & $1.36(0.28)$ \\
FICC (ref=No) & $2.08(0.00)^{*}$ & $2.21(0.00)^{*}$ & $2.41(0.28)$ \\
SHH (ref=male) & $1.92(0.15)$ & $1.23(0.00)^{*}$ & $1.98(0.78)$ \\
MANC (ref=No) & $\cdots$ & $4.01(0.00)^{*}$ & $3.45(0.00)^{*}$ \\
\hline McFadden R ${ }^{2}$ & 0.18 & 0.31 & 0.21 \\
\hline Count R & 0.84 & 0.81 & 0.68 \\
\hline LR & $4935.39(0.00)^{*}$ & $3457.98(0.00)^{*}$ & $2,156.8(0.00)^{*}$ \\
\hline Note: OR means Odd Ratio. LR log-likelihood ratio; Values in parenthesis are probability values; *, **, *** are \\
coefficients that are statistically significant respectively at $1 \%, 5 \%$ and $10 \% ; \cdots$ omitted variable from the model; \\
Source: Authors' computation (2020)
\end{tabular}

the country. In reference to women drawn from poor households, those from wealthy homes [aoR: $1.73, \mathrm{p}=0.00$ ] were significantly more likely to undertake a minimum of four ANC visits. Women drawn from Southern geopolitical zones [aOR: 1.71, $\mathrm{p}=0.00$ ] were significantly more likely to undertake a minimum of four ANC visits. Women who were employed [aOR: $1.40, \mathrm{p}=0.00$ ] were significantly more likely to undertake a minimum of four ANC visits compared to non-working women. Women who were household heads [aOR: 1.92, $\mathrm{p}=0.00$ ] were significantly more likely to report a minimum of four ANC visits when compared to those who were drawn from male-headed households. Women who were financially inclusive [aOR: $2.08, \mathrm{p}=0.00$ ] were more likely to undertake a minimum of four ANC visits compared to those who were not.

\subsection{Place of delivery}

In Table 2, we present the results of the influence of health insurance enrolment and other socio-demographic factors on the place of delivery. Place of delivery (the dependent variable) is a proxy for maternal care utilization. The model is collectively significant as shown by the LR statistic $\left(\mathrm{LR}=3457.98\right.$. The count $\mathrm{R}^{2}$ is approximated to 0.81 which implies that health insurance enrolment and other socio-demographic factors collectively account for $81 \%$ of the variation in place of delivery. Health insurance enrolment [aOR: 1.42, $\mathrm{p}=0.00$ ] significantly increase the odds for facility delivery by $42 \%$. Women who reported formal education [aoR: $3.00, \mathrm{p}=0.00$ ] were approximately three times significantly more likely to deliver in health facilities. Women drawn from wealthy households [aOR: 1.96, $\mathrm{p}=0.00$ ] in reference to those from poor households were approximately two times more likely to have their deliveries supervised in health institutions. In reference to those from urban parts, women drawn from rural Nigeria [aOR: $0.77, p=0.00$ ] were less likely to have their deliveries in health institutions. Women drawn from Southern geopolitical zones [aOR: 1.89, $\mathrm{p}=0.00$ ] were more likely to have their deliveries in health institutions. Working women [aOR: 1.23, $\mathrm{p}=0.00$ ], when compared to non-working women, were more likely to have their deliveries in health institutions. Being financially inclusive [aOR: $2.21, \mathrm{p}=0.00$ ] increased the odds for undertaking health facility delivery. Women who were household heads [aOR: $1.23, \mathrm{p}=0.00]$ were significantly more likely to deliver in health institutions. Undertaking a minimum of four ANC visits [aOR: 4.01, $\mathrm{p}=0.00$ ] significantly increased the chances of utilizing facility delivery.

\subsection{Complete child immunization}

In Table 2, we present the results of the influence of health insurance enrolment and other socio-demographic factors on complete child immunization. Complete child immunization (the dependent variable) is a proxy for child care utilization. The socio-demographic variables were used for control purposes. The model is collectively significant as shown by the LR statistic (LR $=2,156.8$. The count $\mathrm{R}^{2}$ is approximated to 0.68 which implies that health insurance enrolment and other socio-demographic factors collectively account for $68 \%$ of the variation incomplete child immunization. Health insurance coverage does not confer any advantage on women to undertake complete child immunization. Women who were enrolled in health insurance 
coverage [aOR: $1.36, \mathrm{p}=0.28$ ] were not significantly different from those who were not under health insurance coverage. In reference to women without formal education, those who reported at least primary education [aOR: 4.32, $\mathrm{p}=0.00$ ] were approximately four times more likely to undertake complete child immunization. Women drawn from wealthy homes [aOR: 4.32, $\mathrm{p}=0.00]$ when compared to those from poor homes were more likely to undertake complete child immunization. Rural women [aOR: $0.46, \mathrm{p}=0.00$ ] were less likely to undertake complete child immunization compared to urban women. Residing in Southern geopolitical zones [aOR: $6.41, \mathrm{p}=0.00$ ] significantly increased the odds for undertaking complete child immunization. Working women [aOR: $1.36, \mathrm{p}=0.00]$ were significantly more likely to undertake complete child immunization when compared to unemployed women. Being financially inclusive [aOR: $1.98, \mathrm{p}=0.00$ ] significantly improved the odds for undertaking complete child immunization. Women who were heads of their homes [aOR: $2.41, \mathrm{p}=0.00$ ] were more likely to undertake complete child immunization compared to those from male-headed households. Women who made up to four ANC visits [aoR: $3.45, \mathrm{p}=0.00$ ] were $245 \%$ significantly more likely to undertake complete child immunization.

\section{Discussion}

In this study, we investigated the impact of health insurance enrolment and selected sociodemographic factors on maternal and childcare utilization in Nigeria. We utilized secondary data from the most recent National Demographic and Health Survey [2018]. The analyses were bounded for 33,173 women who reported recent birth. The results revealed that $57 \%$ of the women reported a minimum of four ANC visits. The coverage rate of a minimum of four ANC visits put at $57 \%$ is low and falls below the rate recoded for several countries in SSA: Zimbabwe [64.8\%]; Lesotho [70.4\%]; Sierra Leone [76.0\%]; Liberia [78.1\%], and Ghana [78.2\%]. Also, $41 \%$ of the women delivered in health institutions. The rate of institutional delivery is below the targeted rate of $100 \%$ [10]. Child immunization coverage rate in this study is put at $27 \%$ and it falls below the $85 \%$ advocated by the expanded programme of immunization. Intervention programme should be implemented in Nigeria that will target the improvement of maternal care behaviour among Nigerian women.

The result from the logistic regression showed that women who were under health insurance were $52 \%$ and $42 \%$ more likely to undertake a minimum of four ANC visits and deliver their babies in health institutions respectively. This finding conforms to the findings from several Ghanaian studies [2,15, 16]. Nigerian study [10], Burkina Faso study [17], Tanzania study [18], for sets of 30 LMICs [19] and Egyptian study [20]. The positive effect of health insurance enrolment on maternal care use recoded in this study is because of health insurance enrolment addresses the issue of affordability and financial barriers to maternal care utilization. It diffuses the financial burden associated with maternal care usage across individuals and overtimes. The study, therefore, advocates for the need to scale up health insurance coverage in Nigeria. Enrolment into the health insurance scheme will enable several women to get rid of the catastrophic and impoverishing effects of the out-of-pocket payment.

The results show that women who had formal education were 170\%, 200\% and 332\% more likely to undertake a minimum of four ANC visits, deliver their babies in health institutions and undertake complete child immunization respectively. This result conforms to findings from several Nigerian studies $[8,13]$, which reported that educated is a marker for various other factors that affect health-seeking behaviour. Dahiru and Ochei [10] hinted that maternal education cofounds with employment opportunities, place of residence, affordability of health bills and women empowerment to influence maternal care behaviour. There is, therefore, a need to expand education opportunities for Nigerian women, particularly those in the rural and Northern part of Nigeria who do not have the opportunity and resources to pursue formal education. At the primary and secondary school levels women should be encouraged using free tuition and at the tertiary level special cut-offs should be used to encourage female school enrolment.

Women who were employed were $40 \%, 23 \%$ and $36 \%$ more likely to undertake a minimum of four ANC visits, deliver their babies in health institutions and undertake complete child immunization. This conforms to findings from past Nigerian study [14]. Women who earn income and exercise control over earned resources will enjoy easy access to healthcare, particularly because they do not depend on their husbands to fund healthcare-related costs. Also, working women are more sociable and mobile as they go outside their homes and can easily contract health information and awareness from colleagues and friends [14].

The result shows that women residing in rural parts of Nigeria were $31 \%, 23 \%$ and $54 \%$ more likely to undertake a minimum of four ANC visits, deliver their babies in health institutions and 
undertake complete child immunization respectively. This finding is in line with several other Nigerian studies $[14,21]$. The disparity in maternal care utilization between rural and urban women suggests contextual factors limiting women's access to modern healthcare services in the rural part of Nigeria. According to Dahiru and Ochei [10], the contextual factors include poverty, inaccessibility of healthcare and ignorance [14]. Therefore, intervention programme design to improve maternal health in Nigeria should focus more on rural women since it may be impossible to improve maternal health statistics for Nigeria without improving it for rural Nigerian women.

We noted that women drawn from Southern geopolitical zones were $71 \%, 89 \%$ and $541 \%$ more likely to undertake a minimum of four ANC visits, deliver their babies in health institutions and undertake complete child immunization respectively. The result conforms to findings from several Nigerian studies [14,21]; however, it contradicts the report by a Nigerian study [10]. A host of factors accounts for the poor utilization of maternal care in Northern parts of Nigeria. First, religious practice does not accommodate elements of women empowerment, but subjects women to their husbands' imperatives. The Purdah tradition encourages female seclusion and limits their access to modern healthcare. Also, poverty and ignorance are higher in the Northern part when compared to the Southern parts of Nigeria.

Also, women drawn from wealthy homes were $73 \%$ and $96 \%$ more likely to undertake a minimum of four ANC visits, and deliver their babies in health institutions respectively. This finding conforms to previous Nigerian studies [10,21,22]; Ghanaian study [23], and Columbian study [24], and Ethiopian study [25]. According to Dahiru and Ochei [10], household socioeconomic status improves both financial and geographical access to health services. Wealth continues to be a significant determinant of maternal care utilization even though several Nigerian States have implemented the policy of free maternal and under-five care [22]. It, therefore, follows that to reduce the effect of poor socioeconomic conditions on maternal and childcare use in Nigeria the government at all levels should go beyond implementing free maternal care to provide other forms of support to women seeking care.

\section{Strength and limitations}

The advantage of the study is that the observation used for the analysis is large being drawn from a nationally representative household survey. However, the following limitations are inherent in the study. First, the analysis undertaken is static hence we could not report the dynamic relationship between health insurance enrolment and maternal/child care behaviour Second, the data analyzed were verbally reported hence may suffer from both recall bias and social desirability bias. Third, the analyses are exclusively for Nigeria as there is no opportunity to extend the findings to Africa countries.

\section{Conclusion}

In this study, we have shown that women who are under health insurance coverage are more likely to complete a minimum of four ANC visits and deliver in allopathic health facilities, where possible complications can be handled by skilled health personnel. However, health insurance coverage has no significant effect on complete child immunization because immunization care is rendered free of charge in most Primary Healthcare Centers (PHCs) in Nigeria. The study revealed a disparity in access to maternal/child care along with the place of residence, region and wealth quintiles. The disadvantaged women are the poor, those in the rural part of Nigeria and the Southern part of Nigeria. Hence, intervention programmes designed to improve women's access to care should focus on women in this part of the country.

\section{Funding}

The study has no funding to report.

\section{Acknowledgements}

The author is grateful to MEASURE DHS for access to the data set used for the analysis in this study. 


\section{Availability of data and materials}

The dataset used and analyzed during the current study is available from the author on a reasonable request.

\section{Disclosure statement}

No potential conflict of interest was reported by the author.

\section{References}

[1] WHO, UNICEF, UNFPA, World Bank, and the United Nations Population Division (2017) Trends in maternal mortality: 2000- 2017 Geneva: World Health Organization.

[2] Yaya S, Da F, Wang R, et al. Maternal healthcare insurance ownership and service utilization in Ghana: Analysis of Ghana Demographic and Health Survey. PLoS ONE, 2019, 14(4): e0214841. https://doi.org/10.1371/journal.pone.0214841

[3] Yaya S, Okonofua F, Ntoimo L, et al. Gender inequity as a barrier to women's access to skilled pregnancy care in rural Nigeria: a qualitative study. International health, 2019, 11(6): 551-560. https://doi.org/10.1093/inthealth/ihz019

[4] Rolle RA and Iseghohi OJ. Predictors of Antenatal Care Utilization in Primary Healthcare Centers in Eight Rural Communities in Delta State, Nigeria. African Journal of Health Economics, 2019, 8(1): $1-22$. https://doi.org/10.35202/AJHE.2019.810122

[5] De Browere V. Strategies for reducing maternal mortality in developing countries: What can we learn from the history of industrialized west? Tropical Medicine and International Health, 1998, 3(10): 771- 782 . https://doi.org/10.1046/j.1365-3156.1998.00310.x

[6] WHO: World Health Report. WHO Geneva, 2000.

[7] Fagbamigbe AF and Idemudia ES. Barriers to antenatal care use in Nigeria: evidence from non-users and implications for maternal health programming. BMC Pregnancy Childbirth, 2015, 15: 95. https://doi.org/10.1186/s12884-015-0527-y

[8] Okonofua F, Ntoimo L, Ogungbangbe J, et al. Predictors of Women utilization of PHC for skilled pregnancy care in rural Nigeria. BMC pregnancy and child, 2018, 18: 106. https://doi.org/10.1186/s12884-018-1730-4

[9] Singh A. Supply-side barriers to maternal healthcare utilization at health sub Centre in India. Peer J, 2016, 4: e2675. https://doi.org/10.7717/peerj.2675

[10] Dahiru T and Oche OM. Determinants of antenatal care, institutional delivery and postnatal care services utilization in Nigeria. Pan African medical journal, 2015, 21: 321. https://doi.org/10.11604/pamj.2015.21.321.6527

[11] Nketiah-Amponsah E and Arthur E. Choice of delivery facility among expectant mothers in Ghana: does access to health insurance matter? Journal of Health Management, 2013, 15(4): 509-524. https://doi.org/10.1177/0972063413516221

[12] Adinma ED and Adinma BDJ. Community-based healthcare financing: An untapped option to a more effective healthcare funding in Nigeria. Nigerian Medical Journal, 2010, 51(3): 95.

[13] National Population Commission N. Nigeria demographic and health survey 2018. Abuja: National Population Commission. 2018: 630.

[14] Ahuru RR. The influence of women empowerment on maternal and childcare use in Nigeria. International Journal of Healthcare Management. 2019, 12: 1-10. https://doi.org/10.1080/20479700.2019.1688505

[15] Nketiah-Amponsah E, Senadza B and Arthur E. Determinants of the utilization of antenatal care services in developing countries. African Journal of Economic and Management Studies. 2013, 4(1): 58-73. https://doi.org/10.1108/20400701311303159

[16] Mensah J, Oppong JR and Schmidt CM. Ghana's National Health Insurance Scheme in the context of the health MDGs: an empirical evaluation using propensity score matching. Health Economics, 2010, 19(Supp 1):95-106. https://doi.org/10.1002/hec.1633

[17] De Allegri M, Ridde V, Louis VR, et al. Determinants of the utilization of maternal care services after the reduction of user fees: a case study from rural Burkina Faso. Health policy, 2011, 99(3): 210-218. https://doi.org/10.1016/j.healthpol.2010.10.010

[18] Kibusi SM, Sunguya BF, Kimunai E, et al. Health insurance is important in improving maternal health service utilization in Tanzania-analysis of the 2011/2012 Tanzania HIV/AIDS and malaria indicator Survey. Bmc Health Services Research, 2018, 18(1): 112. https://doi.org/10.1186/s12913-018-2924-1 
[19] Wang W, Gheda T and Lindsay M. Health Insurance Coverage and Its Impact on Maternal Health Care Utilization in Low-and Middle-Income Countries. DHS Analytical Studies No. 45. Rockville, Maryland, USA: ICF International, 2014

[20] Rashad AS, Sharaf MF and Mansour EI. Does public health insurance increase maternal health care utilization in Egypt? Journal of International Development, 2019, 31(6): 516-520. https://doi.org/10.1002/jid.3414

[21] Okigbo CC and Eke AC. Skilled birth attendance in Nigeria: a function of frequency and content of antenatal care. African journal of reproductive health, 2015, 19(1): 25-33. https://doi.org/10.4314/ajrh.v19i1

[22] Fagbamigbe AF and Idemudia ES. Wealth and antenatal care utilization in Nigeria: policy implications. Health care for women international, 2017, 38(1): 17-37. https://doi.org/10.1080/07399332.2016.1225743

[23] Arthur E. Wealth and antenatal care use: implications for maternal health care utilization in Ghana Health Economic Review, 2012, 2(14): 1-18. https://doi.org/10.1186/2191-1991-2-14

[24] Ortiz AV. Determinants of demand for antenatal care in Columbia. Health Policy, 2007, 86(2-3): 363-372. https://doi.org/10.2139/ssrn. 1537140

[25] Wilunda C, Quagho G, Putoto G, et al. Determinants of Utilization of antenatal care and skilled birth attendant in South West Shoa Zone, Ethiopia: a cross-sectional study. Reproductive Health, 2015, 12 74. https://doi.org/10.1186/s12978-015-0067-y 